\title{
Comparative Vascular Anatomy of the Hip of the Miniature Dog and of the Normal-size Mongrel
}

\author{
KENJI FUJIKAWA \\ Department of Orthopaedic Surgery, Kurume University School \\ of Medicine, Kurume, 830 Japan
}

Received for publication June 5, 1991

\begin{abstract}
Summary: In order to investigate the aetiology of Perthes' disease in the dog the author has conducted a comparative anatomical study of the vascular system of the femoral heads in miniature dogs and in normal-size mongrels. The study was made in five three-months old miniature dogs and five agematched normal-size mongrels, in which the epiphyseal plate of the femoral head was still open. The most distinct difference between the two species was in the channel of the superior retinacular vessels. In miniature dogs, these vessels go through the shallow neck and appear as a "suspended bridge". In normal-size mongrels they go through the deep fossa of the femoral neck and appear to be very rigidly stable. Although foveolar vessels in the round ligament were present in both species, no vessels were found in either species reaching the epiphysis by penetrating the articular cartilage at this stage of development.
\end{abstract}

Key words: Perthes' disease - miniature dog - normal-size mongrel - lateral epiphyseal vessels - superior retinacular vessels

\section{Introduction}

Perthes' disease in man is associated with low birth weight (Molloy, 1967), delayed bone growth and hypoplasty of the femoral head (Goff, 1954; Harrison, 1976). However it is not clear why hypoplastic hips are likely to fail in Perthes' disease.

Moltzen-Nielsen has reported that naturally-occuring Perthes' disease in the dog is seen in only miniature species such as terrier, miniature poodle, and a few others (Moltzen-Nielsen, 1938), and is associated with similar findings as in the man. Should the reason why Perthes' disease in the dog occurs only in miniature species be established, it may give a suggestion to the elucidation of the cause of Perthes' disease in the man.

\section{Materials and Methods}

Five miniature dogs and five normalsize mongrels, all three months old, were investigated. Under either and intravenous barbiturate general anesthesia, a median incision was made over the $a b$ dominal wall in each puppy. The abdominal aorta and inferior vena cava were exposed. A cannula was placed into the aorta. Each animal was sacrificed by perfusing through the inferior vena cava heparinized $0.9 \%$ saline containing $10 \%$ gelatin and India ink (filtered through layers of flannel cloth). About $100 \mathrm{ml}$ of this solution was injected with a syringe until both the gallbladder and lower limbs turned sufficiently black. All animals died intra-operatively during the perfusion under anesthesia. 24 hours later, the hip joints were removed and prepared for 
examination in the following manner: the joints were dipped in celloidin for about 2 months. They were then fixed in $10 \%$ formalin for about 1-2 weeks, and decalcified in $5 \%$ HCL for a few days. The right hip joints were prepared as frontal slices, while the left hip joints were prepared as horizontal slices. The slices (300 $\mu \mathrm{m}$ thick) were clarified with a mixture of methylsalycilate and isosafrol $(3: 1$ $\mathrm{v} / \mathrm{v}$ ) and stained with H.E. for anatomical observation of the vessels in the articular cartilage, epiphyseal plate and the retinacular foveolar parts of the femoral head.

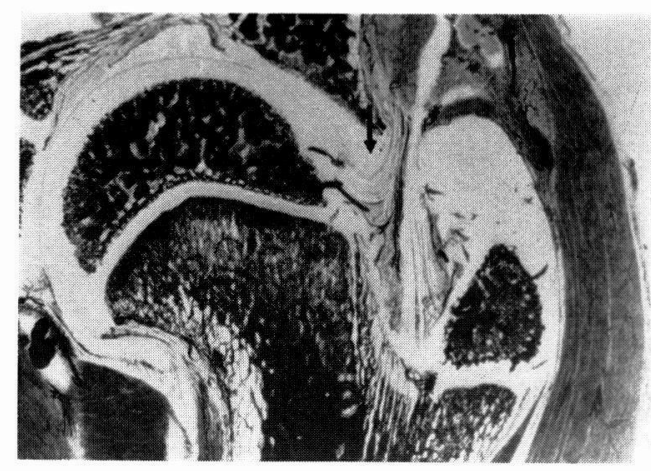

Fig. 1a. Transparent slices obtained from 3-month old miniature dogs, frontal section $\times 5, \quad 300 \mu \mathrm{m}$ thick.

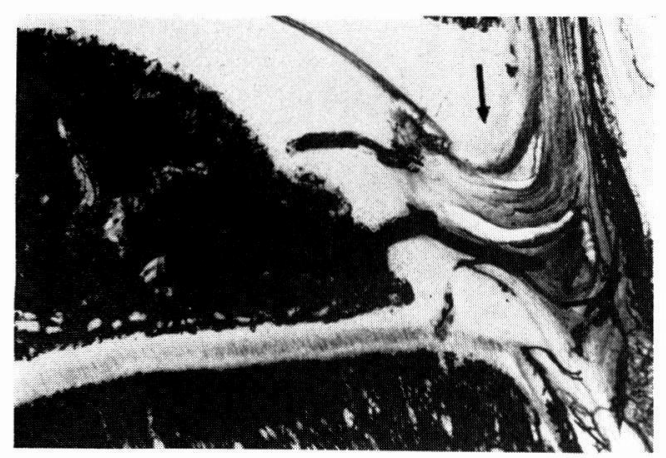

Fig. 1b. The lateral part showing the "suspended bridge" formation with superior retinacular vessels and lateral epiphyseal vessels in a 3-month old miniature dog (arrow).

\section{Results}

\section{1) Lateral part of the hip}

The miniature dogs showed poor growth of the femoral neck and head, a shallow trochanteric fossa (Fig. 1a), and lateral retinacular vessels which were detached from the bony surface, with the appearance of a "suspended bridge" (Fig. 1b).

In contrast in mongrel dogs, the trochanteric fossa showed a sharp U-turn shape with the epiphyseal vessels going along the femoral neck inside the retinaculum. They became the lateral epiphyseal vessels before reaching the epi-

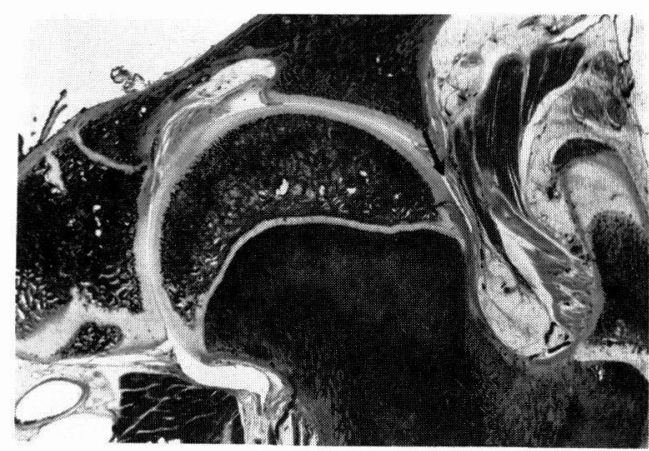

Fig. 2a. Transparent slices obtained from a 3-month old normal-size mongrel, frontal section $\times 5, \quad 300 \mu \mathrm{m}$ thick.

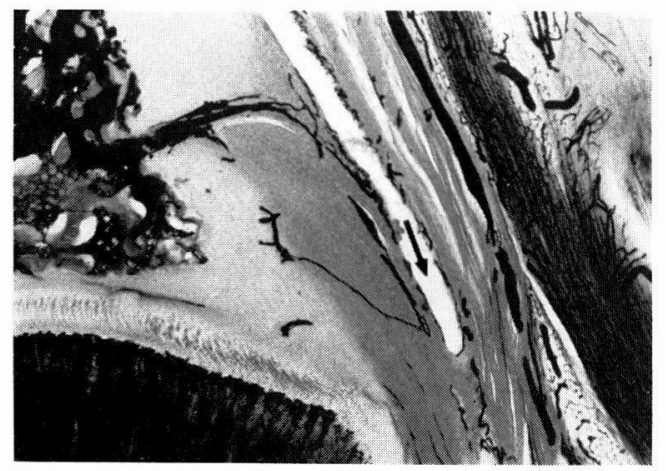

Fig. 2b. The lateral part shows a sharp U-turn of the superior retinacular vessels to the lateral epiphyseal vessels in a 3month old normal-size mongrel (arrow). 
physis (Fig. 2a, b). In the horizontal sections of the femoral head several epiphyseal vessels were seen passing through the chondral tubes between the retinaculum and epiphysis in both species (Fig. 3)

\section{2) Medial part of the hip}

The inferior retinacular vessels in both the mongrel and the miniature dogs were not sufficiently developed for feeding the metaphysis at this stage of development (Fig. 4a, b).

\section{3) Foveolar part of the femoral head}

Penetration of the vessels to the epiphysis through the articular cartilage and the round ligament was not observed in either species (Fig. 5a, b).

\section{4) Epiphyseal plate of the femoral head}

The vascular connection between the epiphysis and metaphysis was interrupted by the epiphyseal plate in both species,

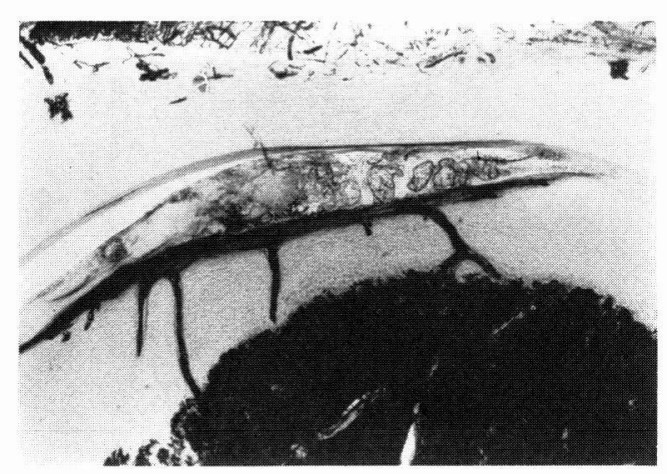

Fig. 3. The lateral part showing several lateral epiphyseal vessels in a miniature dog, horizontal section $\times 5,300 \mu \mathrm{m}$ thick.

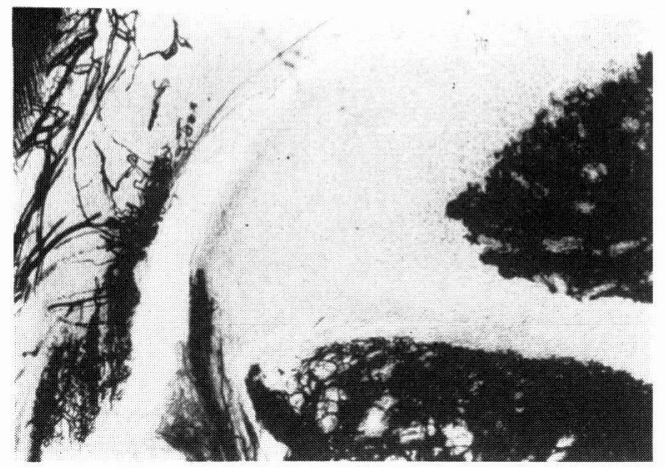

Fig. 4a. Epiphyseal vessels in a medial part are quite scarce in a frontal section in a 3 -month old miniature dog.

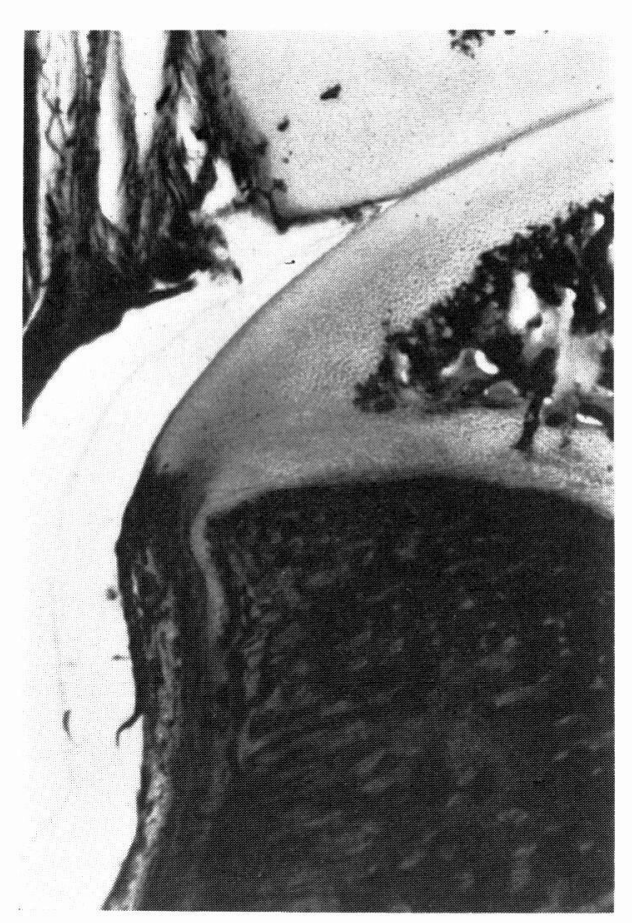

Fig. 4b. No clear-cut epiphyseal vessels exist in a medial part of the femoral in a 3 -month old normal-size mongrel. 
with blood vessels from the epiphysis penetrating to $1 / 3-1 / 2$ depth of the epiphyseal plate (Fig. 6a, b).

The superior retinacular vessels in the miniature dogs gave a branch to the metaphysis before becoming the lateral epiphyseal vessels by penetrating in both the articular cartilage and epiphyseal plate (Fig. 7).

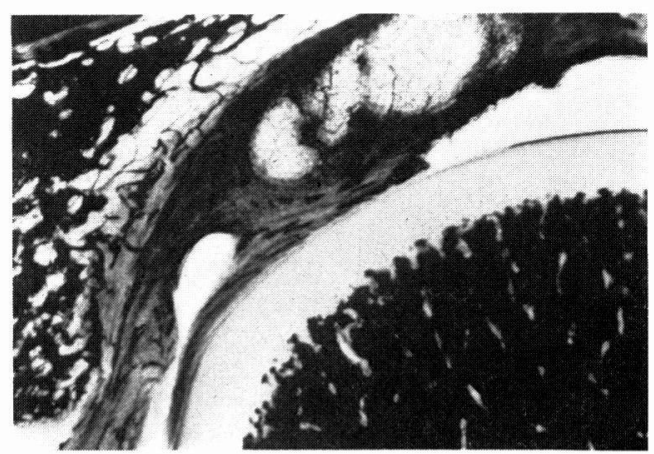

(a)

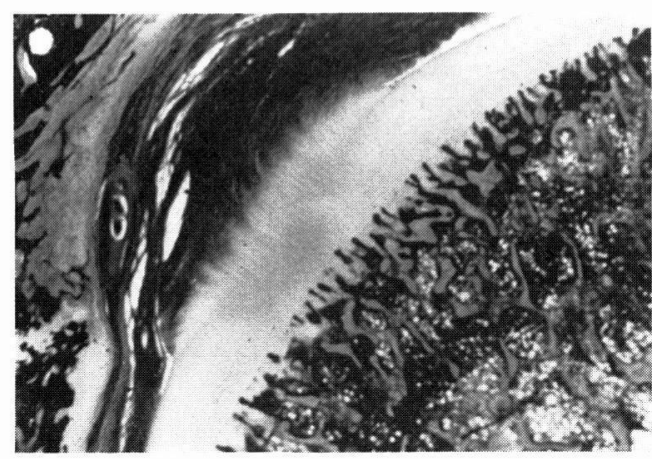

(b)

Fig. 5. The foveolar part in a frontal section in (a) a 3-month old miniature dog. (b) a 3-month old normal-size mongrel. Blood vessels containing India ink were recognized in ligamentum teres, but no communication was noted with the epiphysis.

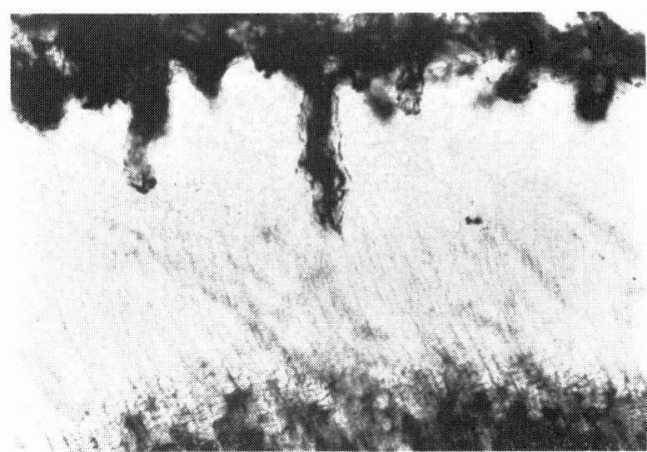

(a)

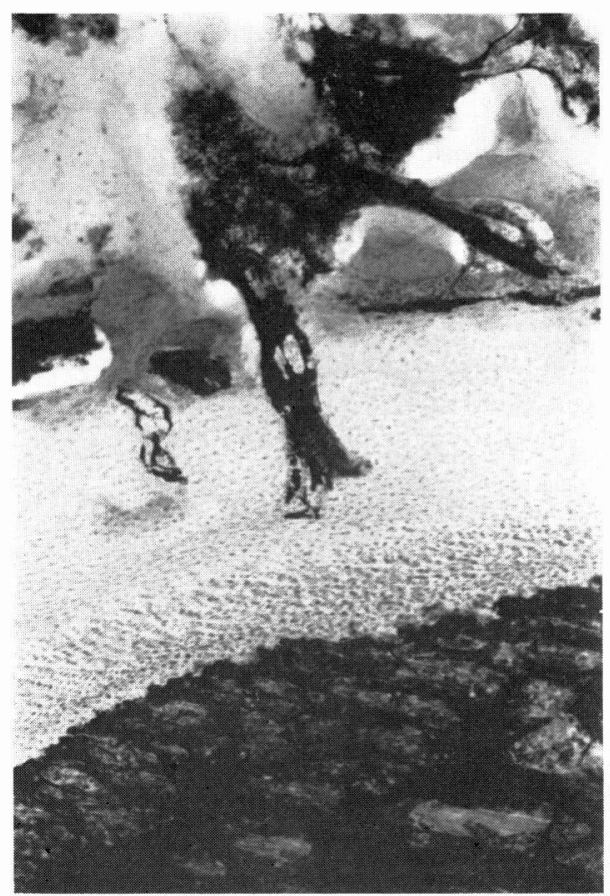

(b)

Fig. 6. Epiphyseal plate in a frontal section in (a) a 3-month old miniature dog. (b) a 3-month old normal-size mongrel. In both sections, the blood vessels go from the epiphysis towards the metaphysis to $1 / 3-1 / 2$ depth into the epiphyseal plate. 


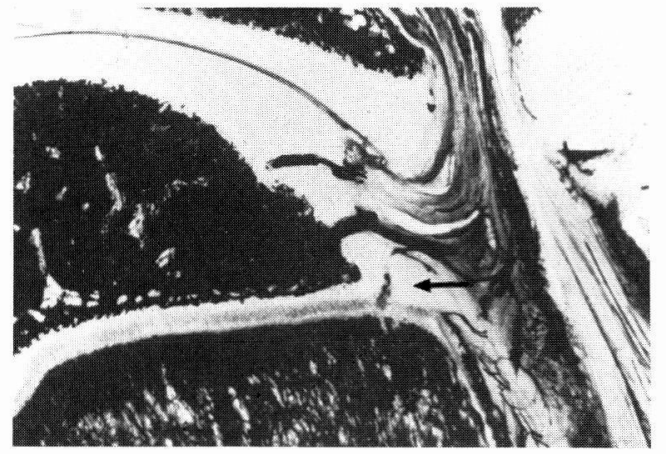

Fig. 7. The lateral part of a frontal section of the femoral head in a 3-month old miniature dog.

Blood vessels go from the superior retinacular vessels towards the metaphysis through the joint cartilage and epiphyseal plate (arrow).

\section{Discussion}

Tucker (1949) gave the first description on the nutritional blood vessels of the human femoral head using a microangiographic technique. Trueta (1957) clarified that the epiphyseal plate played a role as a barrier preventing vascular communication between the metaphysis and the epiphysis. Therefore during the period before the epiphyseal plate closure, the epiphyseal vessels act as a nutritional blood vessels to the femoral epiphysis. This finding suggested that the blood supply to the epiphysis depended on fewer blood vessels from relatively limited sources during the growing period as compared to the nature state.

Crock (1965) showed that there were three major groups of cervical vessels, the anteromedial, the posteromedial, and the lateral. He observed that these vessels entered the hip at an acute angle in the newborn child and that with maturation, as a sequel to differential growth, they became more obtuse in their relation to the developing neck.

Kemp (1973) implicated a transient synovitis in the aetiology of Perthes' disease experimentally induced in Poodle puppies by exposing the cervical vessels to abnormally high intraarticular pressures. He postulated that in the spontaneous disease a transient synovitis and synovial effusion occluded the cervical veins by increasing the intra-articular pressure, thus resulting in ischaemia of the epiphyseal nucleus.

Singleton (1979) suggested that intracapsular tamponade caused compression of the intra-capsular portions of the vessels supplying the capital epiphysis, producing in some cases changes similar to those observed in clinical cases of Perthes' disease. Singleton further discussed the possibility that transient synovitis may be a cause of the intra-capsular vascular obstruction in the pathogenesis of Perthes' disease.

Chung (1976) suggested that the basic extracapsular and transcapsular arterial pattern at the proximal end of the femur was established at birth and persisted throughout life. The lateral ascending cervical vessels provided most of the blood supply to the femoral head and neck. The arteries supplying a large area of the femoral head, neck, and trochanter arose from one single-stem artery which crossed the articular capsule at the posterior trochanteric fossa. Injury to this artery by compression or division may cause significant damage to the bone. Subsynovial ascending cervical arteries have metaphyseal and epiphyseal branches. The epiphyseal arteries pierced the perichondrial ring to supply the ossification center in the femoral head but did not cross the center of the epiphyseal plate in any age group.

Although the importance of the nutritional blood vessels of the femoral head has been discussed by them, no attention has been given the anatomy of the vessels before entering into the femoral head, much less the differential anatomy of 
these vessels between the well developed femoral head and the hypoplastic one.

Meanwhile, it is known that the histology of the femoral head of naturallyoccuring Perthes' disease in the dog was similar to that of Perthes' disease in man (Lee, 1970).

However, there has been no consideration as to why Perthes' disease in the dog occurs only in the miniature species. This comparative study was aimed at clarifing the difference of the vascular anatomy in the hip between the two species, and the following findings were obtained; 1) The femoral head of the miniature dog was smaller than that of the normal-size mongrel, 2) the femoral neck of the miniature dog was shorter, 3) the trochanteric fossa of the miniature dog was shallower, and 4) in mongrels, the nutritional vessels to the femoral epiphysis passed along the deep surface of the trochanteric fossa, while in miniature dogs these vessels passed above the shallow fossa forming a "suspended bridge".

The findings 1)-3) are thougt to be of natural consequence, while the finding 4) indicates a distinct difference in vascular anatomy between the miniature and the mongrel dog. The suspended part of the superior retinacular vessels in the miniature dog may be so exposed as to be susceptible to be damaged.

Atsumi (1986) mentioned from microangiographic studies that Perthes' disease might involve circulatory disturbances in an extracapital area where the superior retinacular artery passed the cervical membrane. Nishio (1974) who studied serial slices of blood vessels in the femoral head of children with Perthes' disease found a lesion in the blood vessels entering from the extracapital area through the cervical membrane.

These facts suggest that the suspended portion of the superior retinacular vessels is likely to be the portion incurring the blood occulsion seen in Perthes' dis- ease.

Although the cause of Perthes' disease is still obscure, this comparative study may be useful for clarifing the pathogenesis of this disease.

Acknowledgments: This study was supported in part by a Grant-in-Aid from the Japan Ministry of Health and Welfare for the Study Group of Idiopathic Aseptic Necrosis of the Femoral Head.

The author thanks Professors A.Inoue and M.Miyazaki for their support and guidance.

\section{References}

Atsumi, K. (1986). Superselective angiography of Perthes' disease. J. Bone Joint Surg. 5, 79-91.

Chung, M.M. (1976). The arterial supply of the developing proximal end of human femur. J. Bone Joint Surg. 58-A, 961-970.

СRоск, H.V. (1965). A revision of the anatomy of the arteries supplying the upper end of the human femur. J. Anat. 99, 77-88.

Goff, Сн. W. (1954). Legg-Calve-Perthes' syndromes and related osteochondroses of youth, ed. Charles C. Thomas, Illinois: Springfield.

HARRISON, M. H. M. (1976). Skeletal immaturity in Perthes' disease. J. Bone Joint Surg. 58-B, $37-40$.

Kemp, H.B.S. (1973). Perthes' disease: An experimental and clinical study. Ann. Roy. Coll. Surg. 52, 18-35.

Lee, R. (1970). A study of the radiographic and histological changes occuring in LeggCalve-Perthes Disease (LCP) in the dog. J. Small Anim. Pract. 11, 621-638.

Molloy, M. K. (1967). Calve-Parthes' disease. J. Bone Joint Surg. 49-A, 498-506.

Moltzen-Nielsen, H. (1938). Cadve-Perthes' Krankheit, Malumdeformans juvenilis coxae bei Hunden. Crch. Wissenschafte U. Pract. Tierhewil. 72, 91-108.

Nishro, A. (1974). Pathological pictures of Perthes' disease in terms of major samples. Orth. Surg. Mook 14, 1-20 (in Japanese).

Singleton, W.B. (1979). The experimental induction of subclinical Perthes' disease in the puppy following arthrotomy and intracapsular tamponade. J. Comp. Path. 86, 57-71. 
TRUeta, J. (1957). The normal vasclar anatomy of the human femoral head during growth. J. Bone Joint Surg. 39-B, 358-394.
Tucker, F.R. (1949). Arterial supply to the femoral head and its clinical importance. J. Bone Joint Surg. 31-B, 82-93. 